\title{
The Effectiveness of Local Gentamicin and Dexamethasone Drops in Posterior Choanoplasty
}

\author{
Bandar Al Qahtani, Yazeed Al Suliman
}

\begin{abstract}
This prospective study is to evaluate the effectiveness of using local gentamicin and steroid drops as a new technique on the success rate of choanal atresia repair in children. The usage of local gentamicin and steroid drops postoperation seems to increase the success rate of posterior choanoplasty. It is a minimally invasive, easily tolerated by both the patients and the parents. It reduces the time of surgery and patients stay in the hospital and reduces the nasal secretion, crustation and adhesion.
\end{abstract}

Keywords: Choanal atresia repair, Posterior choanoplasty, Local gentamicin and local steroid drops, Garasone drops.

How to cite this article: Al Qahtani B, Al Suliman Y. The Effectiveness of Local Gentamicin and Dexamethasone Drops in Posterior Choanoplasty. Clin Rhinol An Int J 2012;5(3): 87-90.

\section{Source of support: Nil}

Conflict of interest: None declared

\section{INTRODUCTION}

Choanal atresia (CA) constitutes a congenital disorder characterized by blockage of the posterior nasal passage usually due to the abnormal presence of bony or membranous tissue. It is the most common nasal congenital disease, affecting approximately 1:5,000 to 8,000 live births and is associated with other abnormalities in $50 \%$ of cases. ${ }^{1}$ CA was first described by Roederer in 1755, and more thoroughly identified by Otto in 1830, while the first report of surgical management was by Emmert in 1853, Flake et al. ${ }^{2}$ CA affects females approximately twice as often as males.

It may appear unilaterally or bilaterally with the former being twice more common than the latter. In $30 \%$ of cases, the obstruction consists solely of bone, while in the other $70 \%$ it is a mixed bony and membranous anomaly. ${ }^{3}$ Congenital bilateral atresia of the posterior nares is a very dangerous disease which can cause death in infants because the inability to nose breathe will lead to tremendous difficulty in sucking and feeding. Mouth breathing in the long term may result in malformation in the midface and/or the hard palate. ${ }^{4}$

The embryologic mechanism seems to be a combination of the persistence of either the nasobuccal membrane of Hochstetler or the buccopharyngeal membrane of the foregut, incomplete resorption of nasopharyngeal mesoderm, and locally misdirected mesodermal flow. This occurs between the fourth and 11th fetal week. ${ }^{5,6}$
Association with other anomalies and syndromes ranges between 20 and 50\%, with no genetic predisposition. The most common syndrome associated with CA is CHARGE. This is an acronym for the set of unusual congenital features, such as coloboma of the eye, heart defects, atresia of the nasal choanae, retardation of growth and/or development, genital and/or urinary abnormalities and ear abnormalities and deafness. ${ }^{7}$

Suspicion for CA arises when $5 \mathrm{~F}$ catheters cannot pass through the nasal cavity into the nasopharynx in neonates. Definitive diagnosis is made with nasal endoscopy. A computed tomographic scan of the sinus region can further demonstrate the atretic plate and assist in identifying anatomy for surgical repair.

Several surgical approaches may be used to treat congenital CA. Historically, the transpalatal technique was the first one described. ${ }^{8}$ Later, laser and endoscopic stenting were used to treat congenital CA. ${ }^{5,9}$ Recently, transnasal endoscopic surgery has developed as a treatment approach for this indication. Powered instruments, such as shavers have been introduced and rendered bony and membranous resection easier. Image-guided surgery allows a better appreciation of the resection needed and increases safety and precision in difficult anatomic cases. ${ }^{10}$ Technical advances and experience in endoscopic nasal surgery have enabled the endonasal endoscopic approach to become the most widely adopted technique nowadays. ${ }^{11}$

\section{MATERIALS AND METHODS}

This is a prospective study approved by the local internal review board and performed on 12 patients with congenital CA (5 bilateral aged 45 to 62 days and 5 unilateral aged 6 to 19 years) were operated upon between 2003 and 2010, 10 females and 2 males, in which all had been referred from Pediatric Hospital in King Saud Medical City. This procedure was done by the same surgeon in our department and the same group of anesthetists. Detailed history and physical examination were obtained and then confirmation was done by computerized CAT scan and all should be checked for CHARGE anomalies workup (echo, U/S abdomen ophthalmologic assessment, ABR and endocrinology referral).

Also, an informed consent form should be signed and the procedure should be done under general anesthesia 
according to the standard preoperative assessment used in our hospital. Exclusion criteria were for those unfit for general anesthesia or with abnormal laboratory results.

No hypotensive technique was used and airway were secured by gauze and neck extended by shoulder roll, $2 \%$ Lidocaine with 1:200,000 epinephrine were applied topically by a pledgelets for 2 minutes at the beginning of the surgery, assessment of the atresia first was checked by catheter then by 0 degree telescope, application of our King Saud Medical City (KSMC). Classification of posterior choana size was graded from I till IV where I complete atresia (0\%), II pinhole choana (less than $10 \%$ ), III small with mucosal band above the floor of the nose (from 10 to $50 \%$ ) and IV wide choana at the level of floor of the nose till basi-sphenoid (from 50 to 100\%). By using endoscopyassisted choanoplasty technique in which perforationdilation technique by different size urethral dilators, the posterior atretic plate perforated and removal of thickened vomer by pediatric back biter was done until a 'neochoana' was created to grade IV on KSMC classification (Figs 1 to 3). No stents or mucoperiosteal flaps were created. All patients received prophylactic systemic antibiotics.

Visual assessment of the newly formed fenestra (neochoana) is done by 0 telescope to assess the diameter and the patency and to check for any complication like perforation of soft or hard palate or CSF leak or active bleeding, if there is any. At the end of the procedure local gentamicin/ steroid drops at a concentration of gentamicin sulfate $0.3 \%$, betamethasone disodium phosphate $0.1 \%$ (trade name Garasone) applied in each nostrils - two drops in each and no endotracheal tubes nasally were used. Patients will be sent to the recovery as KSMC protocols with emphasis on the airway problems-the younger ages will be sent intubated to PICU. Postoperative orders include local

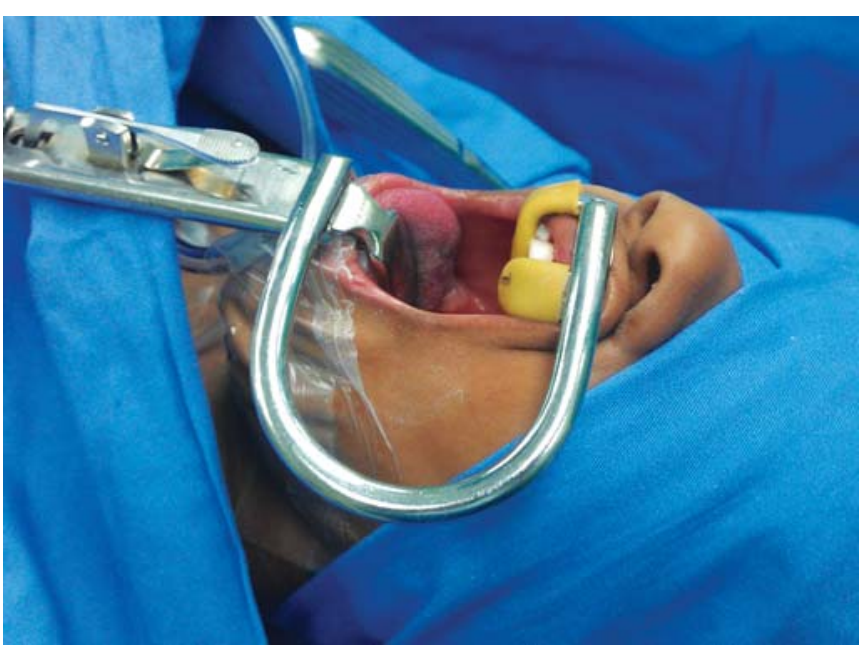

Fig. 1: Position of the patient during the procedure allows good assessment for nasopharynx and the posterior choana, before the usage of perforating dilator

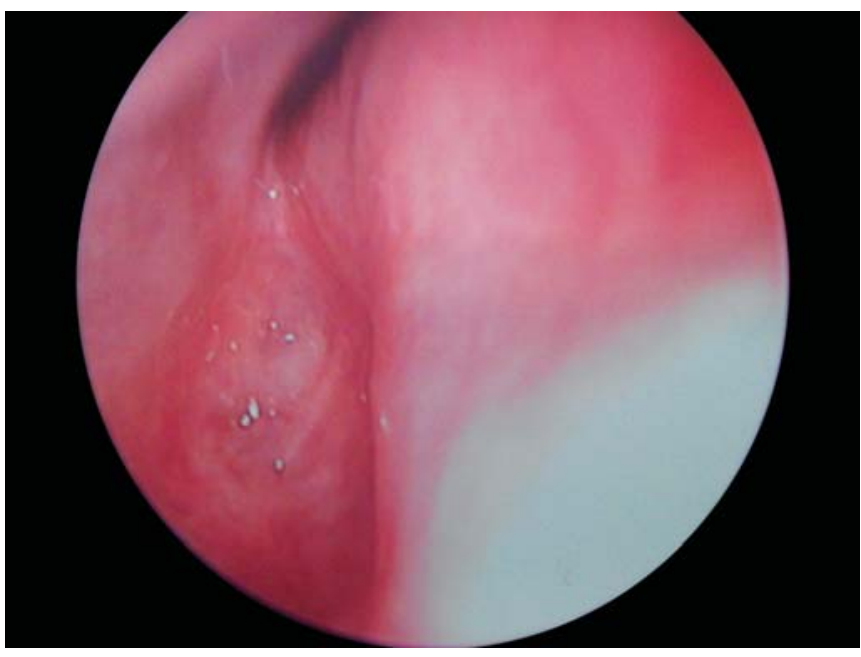

Fig. 2: Endoscopic evaluation of the posterior choana, and here the grade I signifies complete atresia

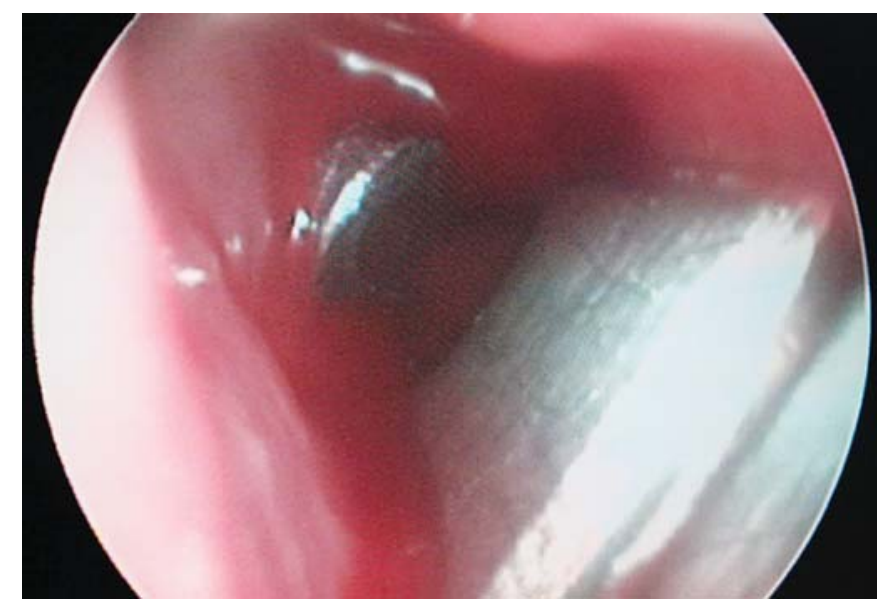

Fig. 3: Back biter is used to remove the thickened vomer and to outward fracturing of inferior turbinate

gentamicin/steroid drops (2 drops/nostril/8h), systemic ABX (2) more dosages, cleansing and suctioning the nose with a soft Foley's catheter (before drops). Patients will be discharged next day-PICU will take 5 more days before discharged as required by ICU doctors. Family strictly were ordered to use the drops regularly (2 drops/nostril/8h) in the first week for the patient older than 5 years and (2 drops/ nostril/12 h) for those younger than 5 and to follow-up weekly in outpatient department OPD with the same doctor. In OPD, 1 week postoperative assessment of the newly formed fenestra by small flexible endoscope size $2.7 \mathrm{~mm}$ visual assessment. Second week dose of local drops will be reduced to two drops/nostril/24 $\mathrm{h}$ and then stopped. Followup from second week will be every month and will be increasing to every 3 months from month 6 .

\section{RESULTS}

Twelve patients underwent posterior choanoplasty (nine patients were new cases and three patients had revision 
repair from previous atresia) surgery between 2003 and 2010. Age of the group ranged from 45 days to 19 years (mean age: 20 years). Ten patients (two males and eight females) fulfilled our criteria and enrolled into the study. The same surgeon and the same anesthetist performed all operations. No major complications were encountered, no intracranial penetration except minor bleeding, two cases stopped by packing with pediatric otrivin drops. The two revisions had a lot of granulation tissues which had been cauterized by silver nitrate sticks then the procedure continued the same like other. Complication encountered during follow-up were as follow: Synechia or adhesion between inferior turbinate and the septum at mid-portion of the turbinate, bleeding in two cases, minor one stopped by topical decongestant, crustation in one case in OPD improve dramatically by next visit, nasal secretion in first day postoperative, two cases in bilateral repair improve next day, (one case did not use the drops), nasal secretion mainly with children younger than 2 months. No other minor or major complication encountered, sleeping disturbance or feeing problems one case, sinusitis one case. In first week, the created fenestrae or the neochoanae were patent and wide assessed by visual estimation got reduced by around 20 to $30 \%$ in first 3 months then to 50\% in 6 months and stopped by that in first 1 year. Two cases had restenosis (one revision case and one new case) (Fig. 4).

There were no problems with the families to comply with the prescribed treatment, except for one patient, where family did not use the drops as somebody advised them not to use it. This case was revision choanoplasty to start with and had to go for another second revision choanoplasty with the same technique as restenosis developed in the first 3 months.

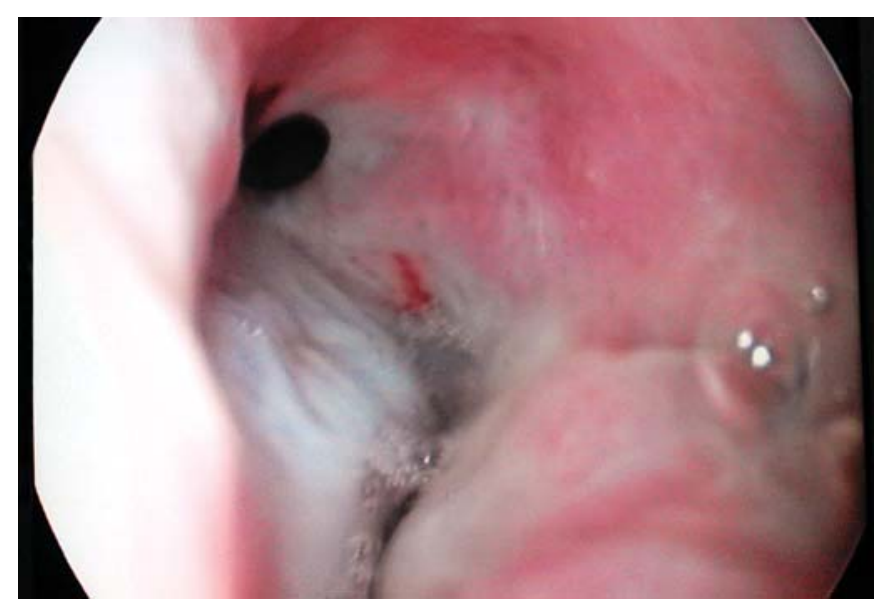

Fig. 4: A case of restenosis and here grade II signifies a pinwhole opening. This patient had problems with sleeping and feeding. Notice the lower lip of the pinwhole raised above the floor of the nose causing retaining of nasal secretion

\section{DISCUSSION}

CA is a challenging condition. Neonates are obligate nasal breathers and may need surgical correction of the stenotic area in the presence of bilateral CA. Goals of the surgical repair include restoring normal nasal passage, preventing further damage to growing structures, and having a short operative time with limited hospitalization afterwards. ${ }^{3}$

As previously mentioned, different surgical techniques have been described for the treatment of CA. The transpalatal approach offers excellent exposure and high success rates. However, increased operative time, bleeding, palatal fistula formation, palatal muscle dysfunction and maxillofacial disturbances are possible sequelae of this procedure thus limiting its popularity. ${ }^{12}$ The transseptal approach offers only narrow exposure and limited possibility to elevate mucosal flaps. It also presents increased risk of eustachian tube or skull base injury. ${ }^{13}$

Endoscopic transnasal repair carries the risk of disruption of growth centers, cerebrospinal fluid leaks, skull base injury and injury to the sphenopalatine artery. ${ }^{5}$ It allows for excellent visualization of the choana, short operative times and very minimal bleeding. Several authors even advocate endoscopic repair in an effort to be able to fashion flaps over raw areas to prevent possible postoperative stenosis. $^{14}$

The use and duration of stenting following surgical management of CA remains under significant debate in the literature. ${ }^{3}$ Most authors agree with the use of a stent for any surgical approach, although exact duration is still controversial, ranging from 4 months ${ }^{15,16}$ to 2 days. ${ }^{17}$

Schoem et $\mathrm{al}^{3}$ was able to show by experience that stenting was not necessary for the successful repair of both unilateral and bilateral CA. This author attributes high success rates without stenting to the evolution of the transnasal approach.

Use of laser in the surgical management of CA has been reported for almost three decades. ${ }^{18,19}$ Many types of laser, including KTP, Nd YAG, contact-diode and carbon dioxide, have been used with good results. ${ }^{18,20}$

The use of mitomycin as an adjunct treatment to CA repair has been explored by some authors. Mitomycin is an aminoglycoside antibiotic made from the fungus Streptomyces caespitosus and has been used as an antineoplastic agent for its ability to inhibit DNA synthesis. Some authors have found that the addition of mitomycin allows for better healing with decreased scar formation in both patients with and without stents. ${ }^{21}$

Our technique, compare to the previously mentioned, is less traumatic, which reduces the granulation tissues, 
possibility of adhesion which may increase the success rate and less risky cooperation of the families were good and noticed as compared to stenting care and it is a cost-effective, quick and safe surgery, less hospital stay and no tubes. We believe that using both the local steroid and local gentamicin play a role and guard against granulation tissues post repair and hence the failure of the procedure.

\section{CONCLUSION}

Choanal atresia can be presented as emergency airway obstruction in bilateral atresia or presented late in life as unilateral atresia. Too many ways for repair done by many surgeons, endoscopic assisted vs. transpalatal, with or without stenting by endotracheal tubes, with or without mitomycin C and with or without mucosal flaps. Success is based on wide removal of vomer bone and to certain extent medial pterygoid plates. By treating and managing granulation tissues helps to reduce the complications. Estimation of the fenestrae or neochoanal size during surgery and afterwards frequent reassessment in the followup, plays a major role in picking up early complication as narrowing of choanae or restenosis. Early application of local gentamicin/dexamethasone drops seems to reduce the granulation tissue dramatically and improves the nasal crustation, edema and secretions. Also it is easy for the family to comply with the drops as compared to the cleansing and suctioning of nasal stenting. It is less invasive technique with less morbidity. It is important to have a good assessment of neochoanae patency and correlate it clinically.

\section{REFERENCES}

1. Panda NK, Simhadri S, Ghosh S. Bilateral choanal atresia in an adult: Is it compatible with life? J Laryngol Otol 2004;118:24445.

2. Flake CG, Ferguson CF. Congenital choanal atresia in infants and children. Ann Otol Rhinol Laryngol 1964;73:458-73.

3. Schoem SR. Transnasal endoscopic repair of choanal atresia: Why stent? Otolaryngol Head Neck Surg 2004;131(4):362-66.

4. Jefferson Y: Mouth breathing: Adverse effects on facial growth, health, academics and behavior. Gen Dent 2010;58:18-25; quiz 26-27, 79-80

5. Samadhi DS, Shah UK, Handler SD. Choanal atresia: A 20year review of medical comorbidities and surgical outcomes. Laryngoscope 2003;113(2):254-58.

6. Dunham ME, Miller RP. Bilateral choanal atresia associated with malformation of the anterior skull base: Embryogenesis and clinical implications. Ann Otol Rhinol Laryngol 1992;101(11):916-19.

7. Gujrathi CS, Daniel SJ, James Al, Forte V. Management of bilateral choanal-atresia in the neonate: An institutional review. Int J Pediatric Otorhinolaryngol 2004;68:399-407.

8. Owens H. Observations in treating seven cases of choanal atresia by the transpalatine approach. Laryngoscope 1951;61(4):304-19.

9. Schwartz ML, Savetsky L. Choanal atresia: Clinical features, surgical approach, and long-term follow-up. Laryngoscope 1986;96(12):1335-39.

10. Schweinfurt JM. Image guidance-assisted repair of bilateral choanal atresia. Laryngoscope 2002;112(11):2096-98.

11. Gosepath J, Santamaria VE, Lippert BM, Mann WJ. Forty-one cases of congenital choanal atresia over 26 years: Retrospective analysis of outcome and technique. Rhinology 2007;45:158-63.

12. Brown OE, Pownell P, Manning SC. Choanal atresia: A new anatomic classification and clinical management application. Laryngoscope 1996;106:97-101.

13. Yaniv E, Hadar T, Shvero J, Stern Y, Raveh E. Endoscopic transnasal repair of choanal atresia. Int $\mathrm{J}$ Pediatric Otorhinolaryngol 2007;71:457-62.

14. Cedin AC, Peixoto Rocha JF Jr, Deppermann MB, Moraes Manzano PA, Murao M, Shimuta AS. Transnasal endoscopic surgery of choanal atresia without the use of stents. Laryngoscope 2002;112(4):750-52.

15. Richardson MA, Osguthorpe JD. Surgical management of choanal atresia. Laryngoscope 1988;98:915-18.

16. Abbeele TVD, Francois M, Narcy P. Transnasal endoscopic treatment of choanal atresia without prolonged stenting. Arch Otolaryngol Head Neck Surg 2002;128:936-40.

17. Sharon-Buller A, Golender J, Savion I, Sela M. Technique for fabrication of splint preventing postsurgical restenosis in choanal atresia. J Prosthetic Dent 2003;90:301.

18. D’Eredita R, Lens MB. Contact-diode laser repair of bony choanal atresia: A preliminary report. Int J Pediatric Otorhinolaryngol 2008 May;72(5):625-28.

19. Healy GB, McGill T, Jako GJ, Strong MS, Vaughan CW. Management of choanal atresia with the carbon dioxide laser. Ann Otol Rhinol Laryngol 1978 Sep-Oct;87(5 Pt 1):658-62.

20. Pototschnig C, Volklein C, Appenroth E, Thumfart WF. Transnasal treatment of congenital choanal atresia with the KTP laser. Ann Otol Rhinol Laryngol 2001 Apr;110(4):335-39.

21. Prasad M, Ward RF, April MM, Bent JP, Froehlich P. Topical mitomycin as an adjunct to choanal atresia repair. Arch Otolaryngol Head Neck Surg 2002;128 (4):398-400.

\section{ABOUT THE AUTHORS}

\section{Bandar AI Qahtani (Corresponding Author)}

Consultant, Department of Otolaryngology, King Saud Medical City Riyadh, KSA, Phone: +966555646405, e-mail: khamsi20@yahoo.com

\section{Yazeed Al Suliman}

Resident, Department of Otolaryngology, King Saud Medical City Riyadh, KSA 